\title{
STM/STS Studies of the Structural Phase Transition in the Growth of Ultra-Thin Bi Films on $\mathrm{Si}(111)$
}

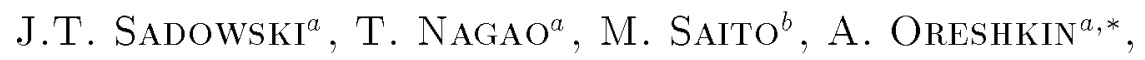 \\ S. Yaginuma ${ }^{a}$, S. HasegaWa ${ }^{c}$, T. Ohno ${ }^{b}$ And T. SakURAI ${ }^{a}$ \\ ${ }^{a}$ Institute for Materials Research, Tohoku University \\ Katahira 2-1-1, Aoba-ku, Sendai 980-8577, Japan \\ ${ }^{b}$ Computational Materials Science Center \\ National Institute for Materials Science \\ 1-2-1 Sengen, Tsukuba, Ibaraki 305-0047, Japan
}

${ }^{c}$ Department of Physics, Graduate School of Science, The University of Tokyo

7-3-1 Hongo, Bunkyo-ku, Tokyo 113-0033, Japan

\begin{abstract}
Growth of thin metal films on semiconductors has been always an important subject for extensive experimental and theoretical studies. As the applicability of well-ordered nanostructures in electronic applications depends strongly on their size and distribution, it is necessary to understand the processes that govern the growth of such structures. In this paper we present the results of investigation of the room temperature growth of thin Bi film on $\mathrm{Si}(111)$. In our study we clarified that rotationally disordered, pseudo-cubic $\operatorname{Bi}\{012\}$ islands with uniform height of $\approx 13 \AA$ are formed in the initial stage of Bi film growth. With increase in the amount of bismuth on the surface, islands interconnect maintaining however their uniform height. This process is further accompanied by the unique and unexpected structural phase transition of the $\{012\}$ film into a hexagonal Bi(001) film.
\end{abstract}

PACS numbers: 68.37.Ef, 68.47.De, 68.55.Ac

\section{Introduction}

The study of the structure and electronic properties of ultra-thin metal films on semiconductor surfaces has always attracted significant attention. Apart of fundamental problems in the physics of thin metallic overlayers such as two-dimensional (2D) electronic transport or quantum confinement effects, also the possible

*Permanent address: Physics Department, Moscow State University, Moscow, 119992, Russia. 
applications for modern nanoelectronics are a driving force for further, more complete research. To establish better control of the crystallinity and electronic properties of the metallic overlayers, it is necessary to understand the factors governing their heteroepitaxial growth, namely, surface free energies and stress relaxation effects. In situ scanning tunneling microscopy/spectroscopy (STM/STS) studies supported by the theoretical calculations can play an important role in increasing our understanding of the processes involved.

It is known that metals grow on the semiconductor surfaces preferentially via the Stransky-Krastanov (S-K) growth mode. Generally, the initial formation of the flat wetting layer is followed by a growth of three-dimensional (3D) islands [1]. Such growth mechanism is thought to be a significant drawback for the realization of flat, well ordered metallic overlayers on semiconductors, suitable for quantum-electronics applications. However, other, unusual growth modes have been also reported in several different epitaxial systems. For example, flat-top 2D islands in $\mathrm{Pb}$ and $\mathrm{Ag}$ growth on silicon $[2,3]$ as well as flat and connected $\mathrm{Mg}$ ultra-thin films on $\mathrm{Si}$ [4] have been recently realized experimentally by employing low-temperature deposition followed by the annealing to room temperature (RT). Currently accepted explanation of these phenomena is based on quantum size effects, particularly the relation between film thickness and the energy of the confined electrons $[5,6]$.

Semimetal Bi has been widely investigated, mostly because of its very interesting electronic properties, such as high carrier mobility, small effective mass, and large Fermi wavelength. Also, recently, extremely high magnetoresistance was observed in electrodeposited Bi thin film and it was attributed to the excellent crystallinity of the film [7]. Bi has metal-like high packing density but its quasi-cubic, layered structure with bilayer stacking is an evidence of covalent-like character of the crystal structure [8]. Immiscibility with $\mathrm{Si}$ makes the $\mathrm{Bi} / \mathrm{Si}$ a convenient system for studying the growth of thin metallic films.

In the present work we discuss the results of the STM/STS studies of the growth of thin bismuth films on $\mathrm{Si}(111)$ substrate. We show that Bi films with nanometer thickness can be grown at $\mathrm{RT}$ on $\mathrm{Si}(111)-7 \times 7$ via $2 \mathrm{D}$ growth mode. However, we propose for this growth a different mechanism than that reported earlier for other epitaxial systems [2-6]. In our experiments we determined that rotationally disordered $B i$ islands with uniform height of $\approx 13 \AA$ are formed in the initial stage of Bi film growth. With increasing the amount of Bi on the surface, islands interconnect keeping their uniform height. This is further accompanied by the unique and rapid structural phase transition of the quasi-cubic $\{012\}$ film into a hexagonal $\mathrm{Bi}(001)$ film.

\section{Experiment}

Bi deposition experiments have been done in the ultra-high vacuum field ion - scanning tunneling microscope (UHV FIM-STM) system, with a base pressure 
below $1 \times 10^{-10}$ Torr. Phosphorus-doped, $n$-type Si(111) wafers, with a resistivity $1.4-1.7 \Omega \mathrm{cm}$ were used as substrates. The $7 \times 7$ reconstruction of the $\mathrm{Si}(111)$ surface was prepared by the resistive flashing of the sample at approximately $1300^{\circ} \mathrm{C}$, followed by short annealing at $830^{\circ} \mathrm{C}$ and slow cooling down to the room temperature. We deposited Bi from the alumina-coated tungsten basket onto the freshly prepared $\mathrm{Si}(111)-7 \times 7$ surface kept at RT. Bi flux was calibrated in situ by observation of the $\mathrm{Bi} \sqrt{3}$ phase formation on $\mathrm{Si}(111)-7 \times 7$, and this calibration was subsequently verified by the ex situ Rutherford backscattering measurements on the thicker Bi layer.

We adopt following convention regarding the $\mathrm{Bi}$ coverage: we define $\mathrm{ML}_{012}$ as the density of $\mathrm{Bi}$ atoms in a pseudo-cubic $\mathrm{Bi}\{012\}$ plane $\left(9.06 \times 10^{14} \mathrm{atoms} / \mathrm{cm}^{2}\right)$ and $\mathrm{BL}_{001}$ as the density in the covalently bonded $\mathrm{Bi}(001)$ bilayer plane $\left(1.14 \times 10^{15}\right.$ atoms $\left./ \mathrm{cm}^{2}\right)$. For reference, the atomic density of a single $\mathrm{Si}(111)$ bilayer $\mathrm{ML}_{\mathrm{Si}(111)}=7.83 \times 10^{14}$ atoms $/ \mathrm{cm}^{2}$. The crystal structure of $\mathrm{Bi}$ can be considered as a slightly distorted simple-cubic lattice where the lattice planes that constitute this pseudo-cube are indexed as equivalent (012), (1112), and (102) (hereafter referred as $\{012\})$. These planes intersect each other with angles of $92.5^{\circ}$ or $87.5^{\circ}$ and the interplane spacing is $3.28 \AA[9]$.

\section{Results and discussion}

In the initial stage of the room temperature $\mathrm{Bi}$ growth on $\mathrm{Si}(111)$ $7 \times 7$ surface a wetting layer consisting of disordered Bi clusters contained within $7 \times 7$ half-unit cells is formed. With $\mathrm{Bi}$ coverage exceeding $0.9-1.1 \mathrm{ML}_{012}$, small nanocrystals start to form on top of the wetting layer, which later leads to the formation of rotationally disordered, tabular islands with uniform height of $\approx 13 \AA$, at coverage exceeding $2 \mathrm{ML}_{012}$. With further increase in the $\mathrm{Bi}$ coverage the islands merge, keeping however their uniform height, which finally results in formation of quite flat, well ordered layer, such as one shown in Fig. 1a. High-resolution STM image taken from that layer (Fig. 1b) shows the periodic structure with rectangular, centered unit cell, with apparent size of $4.6 \pm 0.2 \AA \times 4.8 \pm 0.2 \AA$. The size of the unit cell is consistent with the bulk truncated $\mathrm{Bi}\{012\}$ surface schematically shown in Fig. 1c. Taking this into account, one can easily notice that the observed $\approx 13 \AA$ thickness corresponds exactly to $4 \mathrm{ML}_{012}$. At Bi coverage above $6-7 \mathrm{ML}_{012}$, the structural phase transition from twofold $\{012\}$ film into the threefold $\mathrm{Bi}(001)$ film takes place in the whole volume of the film (Fig. 1d). High-resolution STM image (Fig. 1e) shows hexagonally ordered layer with the nearest-neighbor distance of $4.5 \pm 0.2 \AA$. Together with the observed in the STM images apparent step height of $4.0 \pm 0.2 \AA$, these values correspond very well to bulk values for (001) plane: $4.54 \AA$ and $3.9 \AA$, respectively. Schematic arrangement of the Bi atoms in the (001) plane is shown in Fig. 1f. Please note that the arrows in Fig. 1a and $d$ mark the positions of the underlying Si bilayer steps. 

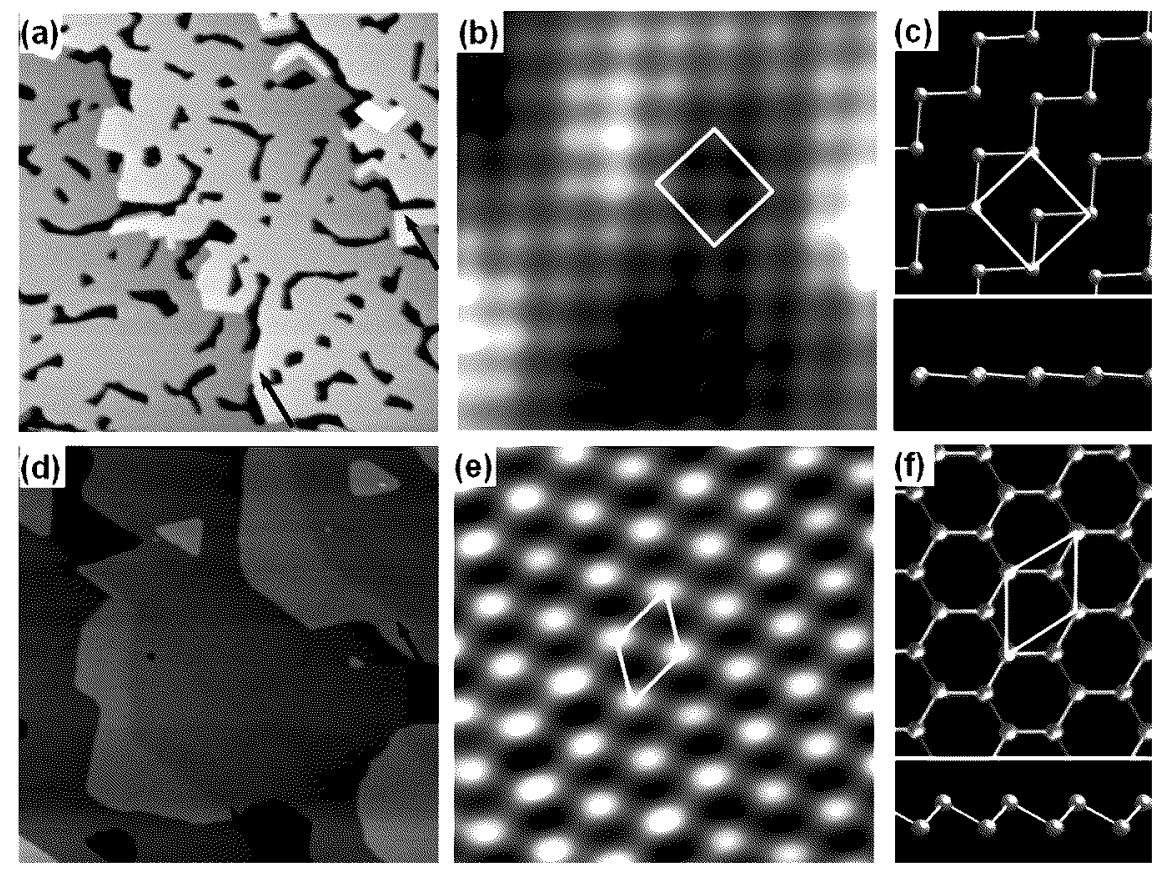

Fig. 1. (a) Topographic STM image $(200 \mathrm{~nm} \times 200 \mathrm{~nm}$, sample bias $+1.8 \mathrm{~V})$ showing merged $\mathrm{Bi}\{012\}$ islands at nominal $\mathrm{Bi}$ coverage of $4.5 \mathrm{ML}_{012}$; (b) high-resolution STM image taken from the layer shown in part (a) - rectangular, centered unit cell with size of $4.6 \pm 0.2 \AA \times 4.8 \pm 0.2 \AA$ is marked; (c) schematic drawing of the bulk truncated Bi\{012\} surface; (d) topographic STM image $(200 \mathrm{~nm} \times 200 \mathrm{~nm}$, sample bias $-2.0 \mathrm{~V})$ taken at nominal $\mathrm{Bi}$ coverage of $11.5 \mathrm{ML}_{012}$, when structural phase transition of the $\{012\}$ film into the $\mathrm{Bi}(001)$ film is completed; (e) high-resolution STM image taken from the surface shown in part (d); (f) schematic arrangement of the Bi atoms in the (001) plane. Arrows in parts (a) and (d) mark the positions of underlying Si steps.

In order to explain observed experimentally formation of rotationally disordered, pseudo-cubic, $\mathrm{Bi}\{012\}$ islands with uniform height, we performed ab initio calculations of the $\mathrm{Bi}\{012\}$ film structure, described elsewhere [10]. Results of the calculations strongly indicated that due to the atomic relaxation, atoms in the top layer of the $\{012\}$ film form new bonds to those in the second layer. As a result, all dangling bonds are saturated. Such relaxation leads to the pairing of the layers, inducing enhanced stability of the $\mathrm{Bi}\{012\}$ film containing even number of layers $(n=2,4,6 \ldots)$. This prediction has confirmed in the experiment where for as-deposited Bi film at this coverage range we observe only 2, 4 (preferentially) and $6 \mathrm{ML}_{012}$ thick $\mathrm{Bi}\{012\}$ islands.

Moreover, calculations indicate also that relaxation of the film by layer pairing is associated with the buckling of the atoms in the top paired layer and the 
corner atoms in the $\{012\}$ unit cell are $\approx 0.5 \AA$ higher than the center atoms. This should result in the inequivalent charge distribution above the center and corner atoms. In Fig. 2a we show the calculated local density of states, integrated from the valence bands covering approximately $0.7 \mathrm{eV}$ below the highest occupied molecular orbital band, which is cut at $\approx 1 \AA$ above the outermost surface layer. The high-resolution, filled state STM image shown in Fig. 2b, taken at sample bias of $-0.7 \mathrm{~V}$ exhibits excellent matching with the charge distribution shown in Fig. 2a. In this image we can only see the corner protrusions in the unit cell. Center protrusion is not visible, which is reflected by high corrugation of $0.4 \pm 0.1 \AA$, observed in the image. From the other side, in the filled state STM image shown in Fig. 2c, taken from $4 \mathrm{ML}_{012}$ high Bi film at sample bias of $-0.016 \mathrm{~V}$ (at this low bias STM tip is much closer to the surface) we can associate observed protrusions with the all $\mathrm{Bi}$ atoms constituting the $\{012\}$ plane. The in-plane lattice constants of $a=4.6 \pm 0.2 \AA$ and $b=4.8 \pm 0.2 \AA$, respectively, are very close to that of bulk terminated $\{012\}$ plane.
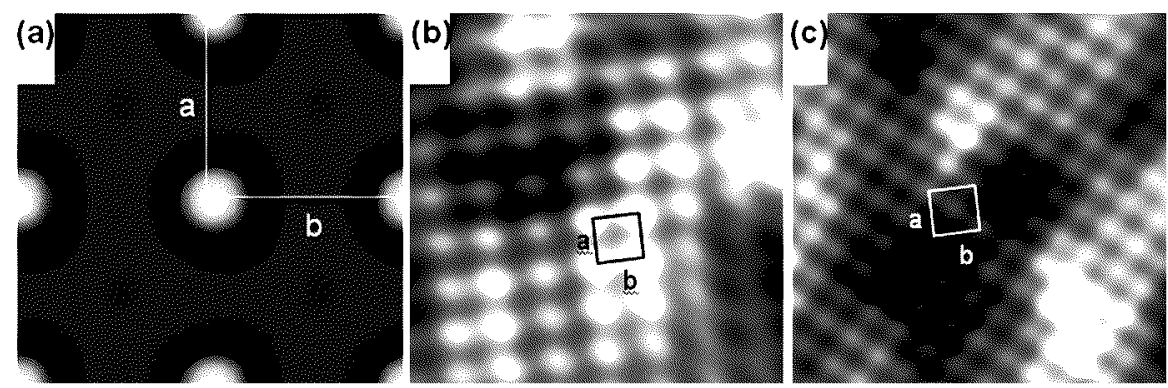

Fig. 2. (a) Calculated local density of states, integrated from the valence bands covering approximately $0.7 \mathrm{eV}$ below the highest occupied molecular orbital band, which is cut at $\approx 1 \AA$ above the outermost surface layer; (b) high-resolution, filled state STM image $(30 \AA \times 30 \AA$ ) taken at sample bias of $-0.7 \mathrm{~V}$ exhibiting excellent matching to the charge distribution shown in Fig. 2a; (c) high-resolution filled state STM image $(30 \AA \times 30 \AA)$, taken low at sample bias of $-0.016 \mathrm{~V}$, showing all atoms constituting $\mathrm{Bi}\{012\}$ surface. In all images the unit cells are outlined by rectangles with $a=4.6 \AA$ and $b=4.8 \AA$, respectively.

We have also compared the calculated partial density of states (PDOS) for the $4 \mathrm{ML}$ high $\{012\}$ layer with the results of our STS experiments. The normalized $(\mathrm{d} I / \mathrm{d} V) /(I / V)$ curve taken with feedback stabilized at sample bias of $-1.2 \mathrm{~V}$ and tunneling current of $0.5 \mathrm{nA}$ is shown in Fig. 3. One can note that the position of the peaks at around $0.1 \mathrm{eV}$ below and above Fermi level observed in the experimental curve (solid line) qualitatively correspond well to the calculated PDOS (dashed line). However, quantitative analysis of the STS spectra still requires finer tuning of our structure model proposed for the $\mathrm{Bi}\{012\}$ film. 


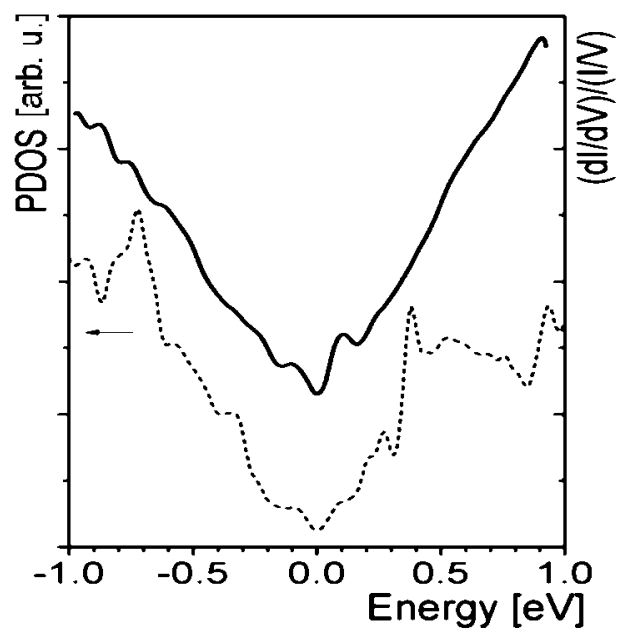

Fig. 3. Normalized $(\mathrm{d} I / \mathrm{d} V) /(I / V)$ curve (solid line) measured from the $4 \mathrm{ML}_{012}$ $\operatorname{Bi}\{012\}$ layer, with feedback stabilized at sample bias of $-1.2 \mathrm{~V}$ and tunneling current of $0.5 \mathrm{nA}$; compared with the calculated PDOS for the $4 \mathrm{ML}$ high $\{012\}$ layer (dashed line).

\section{Summary}

We have presented a novel RT growth of thin Bi films on Si(111) surface. We determined that pseudo-cubic, Bi\{012\} islands with uniform height of about $13 \AA$ are formed in the initial stage growth. With increasing the Bi coverage, islands interconnect keeping their uniform height. Using ab initio calculations we clarified that $\mathrm{Bi}\{012\}$ film undergoes relaxation, which leads to the pairing of the layers, resulting in the enhanced stability of the even-number-layered Bi $\{012\}$ film at RT. Further increase in $\mathrm{Bi}$ amount on the surface is accompanied by the unique and unexpected structural phase transition of the $\{012\}$ film into a well-ordered, hexagonal $\mathrm{Bi}(001)$ film, at nominal coverage of about $8-10 \mathrm{ML}_{012}$.

\section{References}

[1] J. Tersoff, R.M. Tromp, Phys. Rev. Lett. 70, 2782 (1993).

[2] M. Hupalo, V. Yeh, L. Berbil-Bautista, S. Kremmer, E. Abram, M.C. Tringides, Phys. Rev. B 64, 155307 (2001).

[3] L. Gavioli, K.L. Kimberlin, M.C. Tringides, J.F. Wendelken, Z. Zhang, Phys. Rev. Lett. 82, 129 (1999).

[4] L. Aballe, C. Rogero, K. Horn, Phys. Rev. B 65, 125319 (2002).

[5] W.B. Su, S.H. Chang, W.B. Jiang, C.S. Chang, L.J. Chen, T.T. Tsong, Phys. Rev. Lett. 86, 5116 (2001).

[6] Z. Zhang, Q. Niu, C.K. Shih, Phys. Rev. Lett. 80, 5381 (1998). 
[7] F.J. Yang, K. Liu, K. Hong, D.H. Reich, P.C. Searson, C.E. Chien, Science 284, 1335 (1999).

[8] G. Jezequel, J. Thomas, I. Pollini, Phys. Rev. B 56, 6620 (1997).

[9] F. Jona, Surf. Sci. 8, 57 (1967).

[10] T. Nagao, J. T. Sadowski, M. Saitoh, S. Yaginuma, T. Kogure, Y. Fujikawa, T. Ohno, S. Hasegawa, T. Sakurai, submitted to Phys. Rev. Lett. 